\title{
Is Distal Femur Locking Plate a Superior Implant in Distal Femur Fracture?
}

\author{
Anil Chander, Ganesan Ram Ganesan*, Vignesh Jayabalan \\ Department of Orthopaedics, Sri Ramachandra Medical Collage, Chennai, India \\ Email: anil.ortho@gmail.com,
}

Received 1 August 2015; accepted 29 August 2015; published 1 September 2015

Copyright (C) 2015 by authors and Scientific Research Publishing Inc.

This work is licensed under the Creative Commons Attribution International License (CC BY). http://creativecommons.org/licenses/by/4.0/

(c) (i) Open Access

\section{Abstract}

In spite of advances in techniques and improvements in surgical implants, confusion still prevails regarding use of specific implants in distal femur fracture. Fractures in the distal femur have posed considerable therapeutic challenges throughout the history of fracture treatment. Most of these surgical failures were due to inadequate fixation of the fracture fragments. The Aim of the study is to determine whether distal femur locking plates are superior implants than dynamic condylar screw and distal femur nail. A prospective and observational study was done in Department of Orthopaedics and Traumatology, Sri Ramachandra Medical Collage, Chennai between Jan. 2011 and Jan. 2015. Patients with distal femoral fractures, admitted into the hospital, were treated using various modes of internal fixation and followed up over a period of six months to one year and their functional outcome was evaluated. The functional and radiological assessment of patients during follow-up was done using Neer's criteria. The use of any one of the implant, i.e. distal femur locking plates, dynamic condylar screw or distal femur nailing for internal fixation, depends on the type of fracture and the pre operative planning and intra operate decision of the surgeon. In our study, 38\% of fractures treated by Dynamic condylar screw, $\mathbf{7 2 \%}$ of fractures treated by distal femur locking plate and $42 \%$ of fractures treated by Distal femur supracondylar nail had excellent/satisfactory results. Locking plates had better outcome in both extra-articular and intra-articular group. They also had better outcome in both younger and older age groups.

\section{Keywords}

Distal Femur, Supacondylar Femur, Distal Femur Locking Compression Plate, Distal Femur Nail

"Corresponding author. 


\section{Introduction}

Fracture of distal femur accounts for 7 percent of all femoral fractures and excluding hip fractures, it includes 30 percent of fractures of femur [1]. Fractures of distal femur are complex injuries that are difficult to manage and have potential to produce long-term disability. These fractures often are unstable and comminuted and tend to occur in elderly or multiply injured patients. The incidence is highest in females over the age of 75 years and in males between the ages of 15 and 24 years [2]. Because of the proximity of these fractures to the knee joint, regaining full knee motion and function may be difficult. The incidences of malunion, nonunion, and infection are relatively high in many reported series. In older patients, treatment may be complicated by previous joint arthroplasty. In spite of advances in techniques and improvements in surgical implants, confusion still prevails regarding use of specific implants, post operative management and rehabilitation in treatment of these fractures. Long-term disability can still occur in patients with extensive articular cartilage damage, marked bone communition and severe soft tissue injury. The aim of the study is to determine whether distal femur locking plates are superior implants than dynamic condylar screw and distal femur nail.

\section{Materials and Method}

A prospective and observational study was done in Department of Orthopaedics and Traumatology, Sri Ramachandra Medical Collage, Chennai between Jan. 2011 and Jan. 2015. Patients with distal femoral fractures, admitted in the hospital were treated using various modes of internal fixation like dynamic condylar screw, distal femur locking compression plate and distal femur supracondylar nail and followed up over a period of six months to one year and their functional outcome evaluated. From initial radiographs patients were classified according to Muller's classification [3] [4]. The inclusion criteria were distal femur fracture patients between age group 21 - 70 years. The patients were divided into extra-articular group (all Muller classification type A1, A2 and A3) and intra-articular group (types C1 and C2). The exclusion criteria were all B type fractures and type C3 fractures of Muller's classification. Other exclusion criteria were any associated injuries such as tibial plateau fractures, patella fractures and femoral shaft fracture, pathological fractures and compound fractures, congenital or acquired deformities of affected limb and patients with associated preoperative nerve or vascular injury. The functional and radiological assessment of patients during follow up was done using Neer's criteria for functional outcome in patients with operated supra condylar fracture femur [5]. After preoperative preparation patients are taken for surgery. Nonlocking buttress plates are not included in this study. No plates are applied through minimally invasive percutaneous plate osteosynthesis technique. No statistical method was used to analyze the results, as the sample size was small.

The use of any one of the implants like distal femur locking plates, dynamic condylar screw or distal femur supracondylar nailing for internal fixation depends on the type of fracture and the pre operative planning and intra operate decision of the surgeon. Postoperatively patients were mobilized non-weight bearing from 2nd postoperative day. Patients were started on active knee bending depends upon patients pain tolerance. Patients were followed up clinically at first, third, sixth month, one year and then yearly. Follow-up X-rays are taken at the end of 1st, 3rd, 6th month and one year post operatively. Clinically fracture was considered as united if there is no pain at the fracture site during palpation and attempted movements of knee, no local increase in warmth at the fracture site, no pain during attempted weight bearing. Radio logically the fracture was considered united when serial roentgenograms shows bony trabecular crossing the fracture site. Functional grading was made using pain; walking capacity; mobility and work. Radiological grading was done based on varus or valgus deformity; union of fracture; shortening; signs of osteoarthritis. Outcome is said to be excellent when the score is above 85 , satisfactory when between 70 and 85 , unsatisfactory when between 55 and 70 and failure when less than 55. The distribution of fractures whether intra articular or extra articular and number of cases in which distal femur locking compression plate, dynamic condylar screws and distal femur supracondylar nails used were tabulated in Table 1.

\section{Discussion}

Fractures in the distal femur have posed considerable therapeutic challenges throughout the history of fracture treatment. Most of these surgical failures were due to inadequate fixation of the fracture fragments [6]. The prognostic factors for supracondylar fracture included age, intra-articular involvement, methods of treatment, timing 
Table 1. Distribution according to fracture group and implant used.

\begin{tabular}{cccccc}
\hline S. No. & Fracture Group & DCS & DF-LCP & DFN & Total \\
\hline 1 & Extra Articular Group & 12 & 14 & 18 & 44 \\
2 & Intra Articular Group & 14 & 16 & 6 & 36 \\
3 & No. of Patients & 26 & 30 & 24 & 80 \\
\hline
\end{tabular}

DCS: Dynamic condylar screw; DF-LCP: Distal femur locking compression plate; DFN: Distal femur Nail.

of joint motion [7]. There has been no uniform reporting of the results of treatment of distal femur fractures. It is difficult to compare the results of different reported series in literature, because of differences in demographic characteristics and differing fracture characteristics and is further complicated by the use of different classification systems and functional rating systems.

There are 80 patients included in the study, majority of them being young males. There were $69(86.3 \%)$ males and $11(13.7 \%)$ females in this study. The present study does not show a biphasic age distribution of the patient population as is usually seen in studies (Bell et al., 1992). Majority of patients belonged to young age group (55\% of patients were 40 years or below). This reflects the mechanism of injury, which was high-energy trauma in $87.5 \%$ of patients most of whom were younger. High male ratio in this study is due to the fact that, males are mainly exposed to high-energy trauma in Indian scenario. The fracture characteristics in our study closely resemble that of study by Bell et al. [8]. High incidence of articular involvement (45\%), fracture communition and associated injuries in these fractures is explained by the fact that high-energy trauma is frequently encountered in these fractures. The relatively low incidence of intra-articular fractures than the extra-articular fractures in this study is due to the fact that type $\mathrm{C} 3$ fractures are excluded from this study, which forms the bulk of intra-articular fractures. Type $\mathrm{C} 3$ fractures were excluded from the study, as retrograde nails are impossible to use/contraindicated in these fractures.

Among the implants used, Dynamic Condylar Screw (DCS) with side plate and Locking Condylar Buttress Plate L-CBP) were included in extra-medullary group and Distal Femoral Nail (DFN) is included in intra-medullary group. Among the extra-medullary group, both DCS and L-CBP are fixed angled devices. Both provide rotational, axial and angular stability. Non-locking simple buttress plates with simple holes are not fixed angled devices and at least theoretically do not provide neither axial nor angular stability. Thus in this study simple buttress plates are not included [9] [10]. Numerous rating scales have been used to determine the functional outcomes after surgical treatment of supracondylar fractures of femur. Neer [5], HSS, Karlstrom and Olerud, Leung, Schatzker, Sanders [11] are some of the rating scales in vogue. We used Neer's scores because it emphasizes on important patient outcome variables such as pain, functions as related to activities of daily living, range of motions, return to work, gross anatomic alignment and roentgen graphic evaluation of union and mechanical alignment. However no rating scale is validated to be superior to other.

Results of our study were comparable with studies by Roby D [12], Mize [13]. In our study, $52.5 \%$ of patients had excellent/satisfactory results and remaining had poor results as evident from Table 2 . In our study, $38 \%$ of fractures treated by DCS, $72 \%$ of fractures treated by L-CBP and $42 \%$ of fractures treated by DFN had excellent/satisfactory results. The results of our series were on a slightly lower side as far as DCS fixation is concerned. This can be attributed to the increased number of deep infections and mal-union rates seen with the implant in our series. Table 3 shows that Extra-articular fracture patients (50\% patients had Neer's score $>70)$ had good results compared to intra-articular fracture patients $(28 \%$ patients had Neer's score $>70)$. Analysing further, DCS had the best results (66\% patients had Neer's score > 70) in young patients with extra-articular fractures. Results were similar (33\% patients had Neer's score $>70)$ in older patients with both extra-articular and intra articular fractures. Worst results were seen in young patients with intra articular fractures $(25 \%$ patients had Neer's score $>70$ ). This can be explained by the severe metaphyseal communition present in this set of patients. Table 4 shows that the average union time of fractures in distal femur locking compression plate group were less compared to dynamic condylar screw group and distal femur supracondylar nail group.

In our series, the results of locking plates were comparable to other western series reports and had the best results among the three implant groups. Here again extra-articular patients $(85.7 \%$ patients had Neer's score > 70 ) had good results compared to intra-articular patients (62.5\% patients had Neer's score > 70). Analysing further, all young patients with extra-articular fractures had good results with locking plates. $75 \%$ of young patients 
Table 2. Outcome distribution (measured by Neer's functional criteria).

\begin{tabular}{cccc}
\hline S. No. & Outcome Assessment & No. of Patients with Final Score $>$ 70 & \% of Patients with Final Score $>$ 70 \\
\hline 1 & Excellent & 10 & $12.5 \%$ \\
2 & Satisfactory & 32 & $40 \%$ \\
3 & Unsatisfactory & 26 & $32.5 \%$ \\
4 & Failure & 12 & $15 \%$ \\
\hline
\end{tabular}

Table 3. Outcome according to fracture type and implant used.

\begin{tabular}{cccc}
\hline S. No. & Fracture Type & Implant Used & No. and \% of Patients with Neers Score above 70 \\
\hline \multirow{2}{*}{ Extra Articular Fractures } & DCS & $6(50 \%)$ \\
& & DF-LCP & $12(85.7 \%)$ \\
& \multirow{2}{*}{ Intra Articular Fractures } & DFN & $10(55.5 \%)$ \\
& & DCS & $4(28.5 \%)$ \\
& & DF-LCP & $10(62.5 \%)$ \\
\hline
\end{tabular}

DCS: Dynamic condylar screw; DF-LCP: Distal femur locking compression plate; DFN: Distal femur Nail.

Table 4. Outcome on the basis of union time and time of weight bearing.

\begin{tabular}{ccccc}
\hline S. No. & Implant Used & $\begin{array}{c}\text { Average Union Time } \\
\text { (in Months) }\end{array}$ & $\begin{array}{c}\text { Average Time to Start } \\
\text { Active Physiotherapy }\end{array}$ & $\begin{array}{c}\text { Average Time to Full Weight } \\
\text { Bearing (in Months) }\end{array}$ \\
\hline 1 & DCS & 6.0 & 1 \& 1/2 Months & 5.0 \\
2 & DF-LCP & 3.5 & 20 Days & 4.0 \\
3 & DFN & 4.5 & 1 Month & 4.5 \\
\hline
\end{tabular}

DCS: Dynamic condylar screw; DF-LCP: Distal femur locking compression plate; DFN: Distal femur Nail.

with intra articular fractures had good results. Among the twenty five percent, bad results were mainly due to fixation failure which is mainly attributed to improper surgical technique. In our study, locking plates had better results compared to other implant groups in older patients. This is in accordance the bio mechanical study by Marti et al. in which he showed had locking plates provide good stability in bone models with poor bone stock [14]. Again, in older patients, extra-articular (67\% patients had Neer's score $>70)$ had better results than intra articular (50\% patients had Neer's score $>70)$ fractures in L-CBP fixation.

Highest average range of movement, union time, time to full weight bearing and start of active physiotherapy is seen in locking plate group, followed by DFN group and DCS group. In our study, among the patients where active exercises started within 1 month, $70 \%$ had good results whereas in patients where active exercises started after 1 month, only $38 \%$ had good results. This apparently large difference is also due to the fact that, those patients in whom physiotherapy was started after 1 month had associated complications, which lead to bad results in them. Nonunion in the distal third of femur is and should be relatively rare for the bone is primarily cancellous and has an excellent vascular supply with good local osteogenic properties. The incidence of this complication seems to vary according to the method with which the fracture was treated. In two large series Neer et al. [5]; Butler et al. [15] involving both open and closed methods of treatment, there was nonunion in 19 of 315 $(6.5 \%)$ and 16 of $110(15 \%)$ cases respectively. Therefore all forms of treatment have had nonunion as a significant complication. In our study, average non-union rate was highest in DCS group (11.5), followed by DFN group (4.2\%) and L-CBP group (3.3\%).

The significant morbidity resulting from post-operative infection of supracondylar fractures are well documented Moore et al. [7]. Least rate infection was Giles et al. [16] with 5 open fractures of 26 had an infection rate of $0 \%$, followed by Pritchett [17] from Arizona reported a 5.2\% rate and Mize et al. [13] a 6.6\% deep infection rate. In our study, average infection rate was highest in DCS group (8.3\%), followed by L-CBP group (6.3\%) as tabulated in Table 5. There were no deep infections in DFN group, as most of the cases were done by closed means. Highest number of secondary surgical procedures was done in DFN group. Four patients needed implant 
Table 5. Complications.

\begin{tabular}{ccccc}
\hline S. No. & Complication & DCS & Locking CBP & DFN \\
\hline 1 & Non Unions & $2(6.5 \%)$ & $1(3.3 \%)$ & $1(4.2 \%)$ \\
2 & Fixation Failure & $3(11.5 \%)$ & $4(13.3 \%)$ & $1(4.2 \%)$ \\
3 & Deep Infections & $2(8.3 \%)$ & $2(6.6 \%)$ & $0(0 \%)$ \\
4 & Secondary Surgical Procedures & $3(11.5 \%)$ & $3(10 \%)$ & $8(24 \%)$ \\
\hline
\end{tabular}

removal following complains of knee pain mainly due to implant protrusion and impingement in knee joint. Four patients needed dynamisation later in follow up to achieve union.

\section{Conclusion}

Locking condylar plate group had the best functional outcome followed by DFN group and DCS group. Locking plates had better outcome in both extra-articular and intra-articular group. They also had better outcome in both younger and older age groups. DFN group had moderately good outcome in extra-articular group, but had the worst outcome in intra articular fractures, thus contraindicated in these fractures. Thus, we can conclude that locking plates remain the first choice in both intra articular and extra articular fractures, in both young and old patients. Next to locking plates, DFN may be used in preference to DCS in extra articular fractures and DCS in preference to DFN in intra articular fractures.

\section{Acknowledgements}

Nil.

\section{References}

[1] Kannus, P., Parkkari, J., Sievänen, H., et al. (1996) Epidemiology of Hip Fractures. Bone, 18, 57S. http://dx.doi.org/10.1016/8756-3282(95)00381-9

[2] Dy, C.J., McCollister, K.E., Lubarsky, D.A. and Lane, J.M. (2011) An Economic Evaluation of a Systems-Based Strategy to Expedite Surgical Treatment of Hip Fractures. The Journal of Bone \& Joint Surgery, 93, 1326-1334. http://dx.doi.org/10.2106/JBJS.I.01132

[3] Müller, M.E., Nazarian, S., Koch, P. and Schatzker, J. (1990) The Comprehensive Classification of Fractures of Long Bones. Springer, Berlin. http://dx.doi.org/10.1007/978-3-642-61261-9

[4] Müller, M.E., Allgöwer, M., Schneider, R. and Willenegger, H. (1979) Manual of Internal Fixation. Springer, Berlin. http://dx.doi.org/10.1007/978-3-642-96505-0

[5] Neer II, C.S., Grantham, S.A. and Shelton, M.L. (1967) Supracondylar Fracture of the Adult Femur. The Journal of Bone \& Joint Surgery, 49A, 591-613.

[6] Mize, R.D., Bucholz, R.W. and Grogan, D.P. (1982) Surgical Treatment of Displaced Comminuted Fractures of the Distal End of the Femur. The Journal of Bone \& Joint Surgery, 64A, 871-879.

[7] Moore, T.J., Watson, T., Green, S.A., Garland, D.E. and Chandler, R.W. (1987) Complications of Surgically Treated Supracondylar Fractures of the Femur. Journal of Trauma-Injury Infection \& Critical Care, 27, 402-406. http://dx.doi.org/10.1097/00005373-198704000-00010

[8] Bell, K.M., Johnstone, A.J., Court-Brown, C.M. and Hughes, S.P.F. (1992) Primary Knee Arthroplasty for Distal Femoral Fractures in Elderly Patients. The Journal of Bone \& Joint Surgery, 74B, 400-402.

[9] Markmiller, M., Konrad, G. and Südkamp, N. (2004) Femur-LISS and Distal Femoral Nail for Fixation of Distal Femoral Fractures: Are There Differences in Outcome and Complications? Clinical Orthopaedics and Related Research, 426, 252-257.

[10] Christodoulou, A., Terzidis, I., Ploumis, A., Metsovitis, S., Koukoulidis, A. and Toptsis, C. (2005) Supracondylar Femoral Fractures in Elderly Patients Treated with the Dynamic Condylar Screw and the Retrograde Intramedullary Nail: A Comparative Study of the Two Methods. Archives of Orthopaedic and Trauma Surgery, 125, 73-79. http://dx.doi.org/10.1007/s00402-004-0771-5

[11] Sanders, R., Swiontokowski, M., Rosen, H. and Helfet, D. (1991) Double Plating of Comminuted Unstable Fractures 
of the Distal Part of the Femur. The Journal of Bone \& Joint Surgery, 73A, 341-346.

[12] Roby, D. and Bucholz, R. (1989) Management of Complex Fractures of the Distal Femur. Clinical Orthopaedics and Related Research, 240, 77-86.

[13] Mize, R.D., Bucholz, R.W. and Grogan, D.P. (1982) Surgical Treatment of Displaced Comminuted Fractures of the Distal End of the Femur. The Journal of Bone \& Joint Surgery, 64A, 871-879.

[14] Marti, R.K., Schuller, H.M. and Raaymakers, E.L. (1989) Intertrochanteric Osteotomy for Non-Union of the Femoral Neck. The Journal of Bone \& Joint Surgery (British Volume), 71, 782-787.

[15] Butler, M.S., Brumback, R.J., Ellison, T.S., Poka, A., Bathon, G.H. and Burgess, A.R. (1991) Interlocking Intramedullary Nailing for Ipsilateral Fractures of the Femoral Shaft and Distal Part of the Femur. The Journal of Bone \& Joint Surgery, 73A, 1492-1502.

[16] Giles, J.B., Delee, J.C., Heckman, J.D. and Keever, J.E. (1982) Supracondylar-Intercondylar Fractures of the Femur Treated with a Supracondylar Plate and Lag Screw. The Journal of Bone \& Joint Surgery, 64A, 864-870.

[17] Pritchett, J.W. (1984) Supracondylar Fractures of the Femur. Clinical Orthopaedics and Related Research, 184, 173177. http://dx.doi.org/10.1097/00003086-198404000-00027 